Gut and Liver, Vol. 10, No. 4, July 2016, pp. 611-616

\title{
Potential Efficacy of Pegylated Interferon- $\alpha$ and a Nucleos(t)ide Analogue as Combination Therapy for HBeAg-Positive Chronic Hepatitis B
}

\author{
Chung-II Wi ${ }^{1}$, W. Ray Kim ${ }^{1,2}$, John B. Gross, Jr. ${ }^{1}$, Linda M. Stadheim ${ }^{1}$, and John J. Poterucha ${ }^{1}$ \\ ${ }^{1}$ Division of Gastroenterology and Hepatology, Mayo Clinic College of Medicine, Rochester, MN, and ${ }^{2}$ Division of Gastroenterology and \\ Hepatology, Stanford University School of Medicine, Stanford, CA, USA
}

See editorial on page 497.

Background/Aims: Despite the potent suppression of the hepatitis $B$ virus with modern antiviral agents, only a minority of HBeAg-positive patients achieve hepatitis B e antigen seroconversion. We aimed to explore the potential efficacy of combination therapy consisting of pegylated interferon (p-IFN) and an oral antiviral agent in patients with HBeAgpositive chronic hepatitis B. Methods: The treatment protocol consisted of p-IFN- $\alpha-2 a$ at $180 \mu \mathrm{g} /$ wk for 48 weeks, with either entecavir or tenofovir added 8 weeks after the initiation of p-IFN and continued for at least 6 months after HBe seroconversion was achieved. Results: To date, 10 patients have been treated under the protocol (eight adults, mean age $36 \pm 8$ years; two adolescents, aged 12 and 16 years). All eight adult patients experienced loss of HBeAg at a mean of $72.3 \pm 66.9$ weeks, including six patients who also developed anti-HBe and one patient who had HBs seroconversion. Although both adolescents remain on therapy, one adolescent had $\mathrm{HBs}$ seroconversion without $\mathrm{HBe}$ seroconversion. A total of nine of our 10 patients experienced a favorable serological transition. Conclusions: The combination of p-IFN and a modern oral antiviral agent may be more effective than monotherapy with either class of agent in the treatment of HBeAg-positive chronic hepatitis B patients. (Gut Liver 2016;10:611-616)

Key Words: Combination antiviral therapy; HBeAg-positive chronic hepatitis B; HBe seroconversion; Pegylated interferon; Entecavir or tenofovir

\section{INTRODUCTION}

Hepatitis B virus (HBV) infection remains an important global health problem. Chronic HBV infection affects more than 360 million people world-wide. In the United States, an estimated 2 million Americans have chronic HBV infection. ${ }^{1}$ The burden of chronic HBV infection results from progressive liver disease that may lead to cirrhosis, end-stage liver disease and hepatocellular carcinoma (HCC).

Currently, there are seven therapeutic agents that have been approved for the treatment of HBV infection, including two interferon- $\alpha$ preparations and five oral nucleoside or nucleotide analogs (NUCs). Of the latter, modern agents such as entecavir and tenofovir possess potent antiviral activities, commonly leading to disappearance of viral genome in the patient serum. However, serological changes indicative of meaningful alterations in the immune response against HBV do not necessarily accompany even the most profound viral suppression. For example, in HBeAg-positive patients, oral NUCs afford less than $50 \%$ chance of HBe seroconversion, even after 5 years of successful antiviral therapy. ${ }^{2-4}$ Results of interferon therapy are not much superior to NUCs with regard to HBe seroconversion. ${ }^{5,6}$

The combination between interferon and NUCs has a theoretical advantage of the two drugs working through different mechanisms, potentially yielding additive or synergistic effects. However, randomized trials to date have not shown the combination therapy to be more advantageous over either agent alone. ${ }^{7-9}$ Unfortunately, those studies were limited by at two aspects of the study design. First, most of the studies used lamivudine, which is well recognized as a suboptimal option given its modest antiviral potency and a low genetic barrier to resistant mutations. Second, in those studies, both interferon and lamivudine were given for the same finite duration such

Correspondence to: W. Ray Kim

Division of Gastroenterology and Hepatology, Stanford University School of Medicine, 300 Pasteur Dr, Always M211, Stanford, CA 94304, USA

Tel: +1-650-725-6511, Fax: +1-650-723-5488, E-mail: wrkim@stanford.edu

Received on July 10, 2014. Revised on September 27, 2014. Accepted on October 9, 2014. Published online July 21, 2015

pISSN 1976-2283 eISSN 2005-1212 http://dx.doi.org/10.5009/gnl14256

@ This is an Open Access article distributed under the terms of the Creative Commons Attribution Non-Commercial License (http://creativecommons.org/licenses/by-nc/4.0) which permits unrestricted non-commercial use, distribution, and reproduction in any medium, provided the original work is properly cited. 
that lamivudine was discontinued before the desired end point (e.g., HBe seroconversion) was reached..$^{10}$ In this manuscript, we report the potential efficacy of combination therapy consisting of pegylated interferon (p-IFN) and a modern antiviral NUC in 10 patients with $\mathrm{HBeAg}$-positive chronic hepatitis $\mathrm{B}$ cared for at Mayo Clinic.

\section{MATERIALS AND METHODS}

The Mayo combination therapy protocol was implemented in 2006 for patients with chronic hepatitis B. This is a management protocol utilizing two approved medications offered to patients as a part of practice, rather than a research protocol. For the purpose of this report, an approval from the Institutional Review Board of Mayo Foundation was obtained for review of the records and publication of the data.

Initially, the protocol was conceived for both HBeAg-positive and -negative patients. However, discouraging early results in the HBeAg-negative patients soon led to the protocol being exclusively applied in HBeAg-positive patients. The protocol

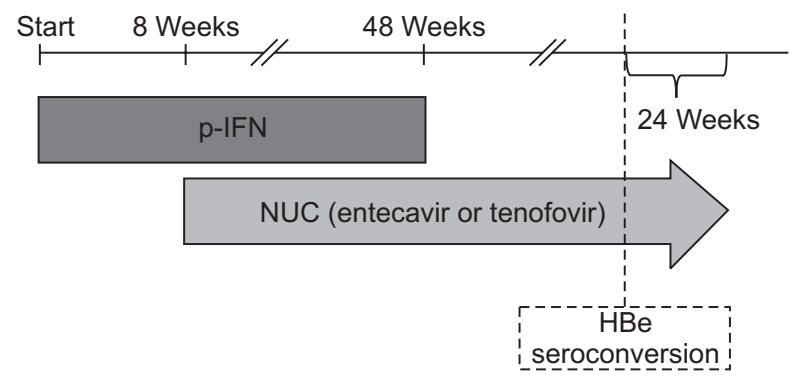

Fig. 1. Schematic illustration of the combination therapy regimen. p-IFN, pegylated interferon; NUC, nucleos(t)ide analogue. consists of p-IFN- $\alpha$-2a $180 \mu \mathrm{g} / \mathrm{wk}$ intended for a course of 48 weeks. At 8 weeks after the initiation of p-IFN, a NUC (entecavir or tenofovir) was added to the regimen (Fig. 1). The choice of the NUC was mainly dictated by the patient's insurance coverage and formulary availability. The combination was continued for another 40 weeks unless HBe seroconversion took place. Following discontinuation of p-IFN at week 48, the NUC was continued until the desired end point was reached, namely $\mathrm{HBe}$ seroconversion. Once HBe seroconversion was achieved, the NUC was continued for a minimum of 6 months before it was discontinued.

Eligibility for the combination therapy protocol included (1) a confirmed diagnosis of chronic HBV infection, including documented HBsAg positivity for at least 6 months; (2) HBeAg positivity; (3) serum alanine aminotransferase (ALT) activities more than 1.5 times the upper limit of normal (ULN); and (4) no evidence of hepatic decompensation. In addition, patients with contraindication for p-IFN, such as significant depression, anemia (hemoglobin $<8 \mathrm{~g} / \mathrm{dL}$ ) or thrombocytopenia (platelets $\left.<50,000 \times 10^{9} / \mathrm{L}\right)$ and planned pregnancy were excluded. Although a liver biopsy was obtained in the majority $(n=9 / 10)$ of the patients, it was not mandatory in the protocol. The results of the biopsy were reported using the Metavir system. ${ }^{11,12}$

Once on therapy, patients were monitored regularly with clinic visits and laboratory follow-up. Supplementary Table 1 summarizes the follow-up schedule of the protocol. Laboratory follow-up included complete blood count, serum concentrations of ALT and HBV DNA, and HBeAg and anti-HBeAg. Standard dose adjustment for p-IFN was instituted in response to cytopenias. For optimal dosing of the NUCs, the manufacturer's package insert was followed including pediatric dosing and renal dose adjustment.

Table 1. Baseline Characteristics

\begin{tabular}{|c|c|c|c|c|c|c|c|c|c|c|}
\hline \multirow{2}{*}{ Characteristic } & \multicolumn{10}{|c|}{ Case no. } \\
\hline & 1 & 2 & 3 & 4 & 5 & 6 & 7 & 8 & 9 & 10 \\
\hline Age/sex & $30 / F$ & $30 / \mathrm{F}$ & $40 / \mathrm{M}$ & $31 / \mathrm{M}$ & $43 / \mathrm{M}$ & $38 / \mathrm{M}$ & $51 / \mathrm{F}$ & $28 / \mathrm{F}$ & $16 / \mathrm{M}$ & $12 / \mathrm{M}$ \\
\hline Race $^{*}$ & 0 & As & As & 0 & C & As & AA & $\mathrm{AA}$ & As & As \\
\hline ALT, U/L & 123 & 146 & 186 & 265 & 117 & 349 & 85 & 86 & 235 & 93 \\
\hline HBV DNA, $\times 10^{6} \mathrm{IU} / \mathrm{mL}$ & $>110$ & 3.4 & $>110$ & 99 & $>110$ & $>110$ & 5.4 & 8.4 & $>110$ & 4.2 \\
\hline Bilirubin, mg/dL & 0.5 & 0.4 & 0.5 & 0.7 & 0.6 & 1.4 & 0.6 & 1 & 0.5 & 0.2 \\
\hline Albumin, g/dL & 4.3 & 4.4 & 3.9 & 4.9 & 4.2 & 4.5 & 4.1 & 3.6 & 4.7 & 4.4 \\
\hline Prothrombin time, sec & 8.1 & 8.5 & 9.5 & 11.1 & 9.6 & 11.3 & 9.5 & 10.4 & 10.1 & 13.6 \\
\hline $\mathrm{Hb}, \mathrm{g} / \mathrm{dL}$ & 13.5 & 14.6 & 13.7 & 15.8 & 14.5 & 14.5 & 14.1 & 13.5 & 15.5 & 13 \\
\hline $\mathrm{WBC}, \times 10^{9} / \mathrm{L}$ & 3.5 & 5 & 6.4 & 8.2 & 5.6 & 4.4 & 5.3 & 4.3 & 8 & 7.7 \\
\hline Platelets, $\times 10^{9} / \mathrm{L}$ & 115 & 265 & 246 & 235 & 256 & 169 & 196 & 153 & 223 & 239 \\
\hline Liver biopsy $^{\dagger}$ & A1F0 & A1F0 & A3F2 & A3F1 & NA & A3F $1-2$ & A1F2 & A3F3 & $\mathrm{A} 2 \mathrm{~F} 1-2$ & $\mathrm{~A} 2 \mathrm{~F} 1$ \\
\hline
\end{tabular}

F, female; M, male; ALT, alanine aminotransferase; HBV, hepatitis B virus; Hb, hemoglobin; WBC, white blood cells; NA, not available.

*Race: 0 (other), As (Asian), C (Caucasian), and AA (African-American); ${ }^{\dagger}$ Histologic scoring system of chronic liver disease (Metavir score). Necroinflammatory score: A0=no activity, A1=mild activity, A2=moderate activity, A3=severe activity. Fibrosis score: $0=$ no fibrosis, $1=$ portal fibrosis without septa, $2=$ few septa, $3=$ numerous septa without cirrhosis, $4=$ cirrhosis. 


\section{RESULTS}

To date, there have been 10 patients with HBeAg-positive chronic hepatitis B treated under the protocol (Table 1). They included eight adults with a mean+standard deviation (SD) age of $36 \pm 8$ years and two adolescents with ages of 12 and 16. Five patients were of Asian ancestry including the two adolescents. As specified in the protocol, all of the patients had elevated serum ALT (>1.5×ULN) with a mean+SD of $195.8 \pm 110.7$ (U/L). Similarly, the serum HBV DNA concentration was quite high with a geometric mean of $6.7 \times 10^{7}(\mathrm{IU} / \mathrm{mL})$. The liver synthetic markers including serum albumin and bilirubin and prothrombin time were within normal limits. Pretreatment blood counts were normal except in case 1, whose white blood cells (WBC) and platelets were slightly below the lower limit of normal. Nine of the 10 patients had a pretreatment biopsies, which showed mostly mild degree of fibrosis with a mean fibrosis score of $1.3 \pm 1.0$ and moderate inflammatory activity with a mean necroinflammatory score of $2.1 \pm 0.9$.

As shown in Table 2, the mean duration of interferon was $39.7 \pm 12.5$ weeks. There were four patients in whom the dura- tion of IFN therapy was shorter than the planned 48 week. In three patients, early HBe seroconversion made it unnecessary to complete the 48 week course. One patient (case no. 8) developed neutropenia, which prompted early termination (please see below for details). In seven patients, entecavir was administered as the oral antiviral, whereas tenofovir was chosen for the remaining three patients. The mean duration of oral antiviral was $90.8 \pm 48.0$ weeks.

All eight out of the eight adult patients (100\%) lost HBeAg, including six patients who also developed anti-HBe, thus achieving HBe seroconversion (75\%). HBe seroconversion occurred after a mean of $72.3 \pm 66.9$ weeks after the initiation of therapy. There was one adult patient (case no. 1) who underwent HBs seroconversion, without anti-HBe being detected. Although neither adolescent has achieved HBe seroconversion and both remain on therapy, one adolescent underwent HBs seroconversion. Thus, altogether, nine of our 10 patients experienced a favorable serological transition (2 HBs seroconversion, $6 \mathrm{HBe}$ seroconversion, and $1 \mathrm{HBeAg}$ loss).

Table 3 describes the current status of the study subjects. As of the last follow-up, three patients remain on treatment (only

Table 2. Details of Treatment and HBe Seroresponse

\begin{tabular}{|c|c|c|c|c|c|c|c|c|c|c|}
\hline & \multicolumn{10}{|c|}{ Case no. } \\
\hline & 1 & 2 & 3 & 4 & 5 & 6 & 7 & 8 & 9 & 10 \\
\hline Duration of IFN, wk & 48 & 48 & 51 & 31 & 48 & 24 & 34 & 16 & 49 & 48 \\
\hline Gap to NUC agent, wk & 9 & 8 & 4 & 8 & 10 & 8 & 8 & 6 & 8 & 8 \\
\hline NUC used* & E & $\mathrm{T}$ & E & E & $\mathrm{T}$ & E & $\mathrm{T}$ & $\mathrm{E} / \mathrm{T}$ & E & E \\
\hline Duration of NUC Tx, wk & 53 & 95 & 128 & 24 & $53^{\dagger}$ & 52 & 126 & $183^{\ddagger}$ & $116^{\dagger}$ & $78^{\dagger}$ \\
\hline Duration of off-treatment follow-up, $\mathrm{wk}^{\S}$ & 18 & 208 & 103 & 35 & - & 15 & 0 & - & - & - \\
\hline HBeAg loss & Yes & Yes & Yes & Yes & Yes & Yes & Yes & Yes & No & No \\
\hline HBe seroconversion & No & Yes & Yes & Yes & No & Yes & Yes & Yes & No & No \\
\hline Time to HBeAg loss or HBe-seroconversion, wk & 62 & 68 & 74 & 8 & 37 & 8 & 105 & 216 & - & - \\
\hline Time to HBV DNA loss, wk & 24 & 20 & 36 & 8 & 37 & 24 & 33 & 36 & 48 & 28 \\
\hline
\end{tabular}

IFN, interferon; NUC, nucleos(t)ide analogue; Tx, treatment; HBV, hepatitis B virus.

${ }^{*} \mathrm{E}$ (entecavir), $\mathrm{T}$ (tenofovir), E/T (entecavir switched to tenofovir); ${ }^{\dagger}$ Therapy ongoing as of the study closure; ${ }^{\mathrm{t}}$ Prophylactic antiviral therapy after liver transplantation excluded; ${ }^{\S}$ Duration from the discontinuation to the last follow-up date.

Table 3. Current Status

\begin{tabular}{|c|c|c|c|c|c|c|c|c|c|c|}
\hline & \multicolumn{10}{|c|}{ Case no. } \\
\hline & 1 & 2 & 3 & 4 & 5 & 6 & 7 & 8 & 9 & 10 \\
\hline Current HBV treatment* & None & None & None & None & $\mathrm{T}$ & None & None & $E^{\dagger}$ & $\mathrm{E}$ & $\mathrm{E}$ \\
\hline HBV DNA, IU/mL & $\mathrm{U}$ & 306 & 557 & 114 & $\mathrm{U}$ & $\mathrm{U}$ & $\mathrm{U}$ & $\mathrm{U}$ & $\mathrm{U}$ & $\mathrm{U}$ \\
\hline $\mathrm{HBeAg}$ & $\mathrm{N}$ & $\mathrm{N}$ & $\mathrm{N}$ & $\mathrm{N}$ & $\mathrm{N}$ & $\mathrm{N}$ & $\mathrm{N}$ & $\mathrm{N}$ & $\mathrm{P}$ & $\mathrm{P}$ \\
\hline $\mathrm{HBeAb}$ & $\mathrm{N}$ & $\mathrm{P}$ & $\mathrm{P}$ & $\mathrm{P}$ & $\mathrm{N}$ & $\mathrm{P}$ & $\mathrm{P}$ & $\mathrm{P}$ & $\mathrm{N}$ & $\mathrm{N}$ \\
\hline HBsAg & $\mathrm{N}$ & $\mathrm{P}$ & $\mathrm{P}$ & $\mathrm{P}$ & $\mathrm{P}$ & $\mathrm{P}$ & $\mathrm{P}$ & $\mathrm{N}^{\dagger}$ & $\mathrm{P}$ & $\mathrm{N}$ \\
\hline ALT, U/L & 23 & 12 & 65 & 23 & 24 & 21 & 28 & 27 & 37 & 38 \\
\hline
\end{tabular}

HBV, hepatitis B virus; $\mathrm{U}$, undetectable $(<100 \mathrm{IU} / \mathrm{mL}) ; \mathrm{N}$, negative; $\mathrm{P}$, positive; ALT, alanine aminotransferase.

${ }^{*} \mathrm{E}$ (entecavir), T (tenofovir); ${ }^{\dagger}$ Post-liver transplantation prophylaxis. 
Table 4. Adverse Event Monitoring (Nadir Lab Values)

\begin{tabular}{|c|c|c|c|c|c|c|c|c|c|c|}
\hline & \multicolumn{10}{|c|}{ Case no. } \\
\hline & 1 & 2 & 3 & 4 & 5 & 6 & 7 & 8 & 9 & 10 \\
\hline $\mathrm{Hb}, \mathrm{g} / \mathrm{dL}$ & 10.5 & 12.2 & 13.6 & 13.1 & 15.2 & 12.8 & 13.4 & 11.4 & 13.3 & 13 \\
\hline PLT, $\times 10^{9} / \mathrm{L}$ & 113 & 167 & 167 & 95 & 36 & 108 & 98 & 73 & 160 & 215 \\
\hline $\mathrm{WBC}, \times 10^{9} / \mathrm{L}$ & 3.1 & 2.7 & 3.9 & 4 & 2.3 & 2.9 & 3 & 2.1 & 3.9 & 4.5 \\
\hline ANC, $\times 10^{9} / \mathrm{L}$ & 1.62 & 0.91 & 1.6 & 1.36 & 0.92 & 0.78 & 1 & 0.47 & 1.33 & 1.59 \\
\hline TSH, mIU/L & 0.1 & 2.1 & 0.8 & 3.7 & 1.9 & 1.1 & 0.4 & 0.4 & 2.4 & 1 \\
\hline
\end{tabular}

$\mathrm{Hb}$, hemoglobin; PLT, platelet; WBC, white blood cells; ANC, absolute neutrophil count; TSH, thyroid stimulating hormone.

oral antiviral), including an adult patient who has lost HBeAg without developing anti-HBe and both adolescents who remain HBeAg-positive. Another patient (case no. 8) developed HCC while on therapy and has undergone liver transplantation since. She is doing well on posttransplant prophylaxis with entecavir without recurrence of HCC or HBsAg. Of patients in whom all therapy was discontinued, three patients became viremic, albeit at very low levels. All three patients retained their HBe seroconverted profile without evidence of a biochemical flare. More detailed description of individual patients is available in Supplementary Data 1 .

Table 4 summarizes safety data. Overall, treatment was well tolerated. There was one patient (case no. 8) whose interferon was discontinued prematurely because of bone marrow suppression. This patient, of African descent, developed neutropenia with an absolute neutrophil count of $470 / \mathrm{mm}^{3}$ at week 16 of p-IFN therapy. Another patient (case no. 1) developed hypothyroidism and was treated with thyroid hormone replacement, which did not affect the course of the scheduled treatment. As expected, both oral antiviral agents, following discontinuation of IFN, were well tolerated.

\section{DISCUSSION}

In this case series, we report the potential effectiveness and safety of a novel regimen using the combination of p-IFN and a modern, "third generation" oral antiviral agent in the treatment of HBeAg-positive chronic hepatitis B patients. In our opinion, it is an important innovation because it resulted in HBe seroconversion or HBeAg loss in all adult patients with less than 2 years of therapy on average. Although the sample size is clearly small, this degree of HBe seroconversion appears much superior to either p-IFN or any of the oral agents alone. The treatment was overall well-tolerated.

Of the classes of antiviral agents currently available for chronic hepatitis B, the advantages of IFN over NUCs include a finite duration of therapy and lack of resistance. In addition, it possesses immunomodulatory effects, in addition to antiviral action, which afford a possibility of immune-mediated clearance of hepatitis B. ${ }^{13}$ On the other hand, modern NUCs, includ- ing entecavir and tenofovir, have proved to be highly potent against HBV, resulting in profound viral suppression virtually without a concern for antiviral resistance. While current guidelines recommend IFN or a NUC for first-line therapy for HBeAgpositive patients, either is less than ideal from the standpoint of achieving HBe seroconversion. ${ }^{14-16}$ Even after prolonged therapy (in the case of a NUC) or follow-up (after IFN), HBe seroconversion occurs in less than $50 \% .^{2-4}$

In addressing this gap, previous investigations have considered combination therapy between interferon and a NUC. ${ }^{7-9}$ There have been at least three large randomized controlled trials evaluating combined p-IFN and lamivudine for HBeAg-positive patients. In all studies, patients were treated for 1 year, and serological, biochemical, and virological responses were compared between the groups of combination therapy and monotherapy of p-IFN or lamivudine at the end-of-treatment and 24 weeks thereafter. The rate of HBe seroconversion was 27\%-36\%, 29\%$32 \%$, and $14 \%-19 \%$ for the combination treatment group, the p-IFN monotherapy group, and the lamivudine monotherapy group, respectively. While they failed to demonstrate superiority of combination therapy, they indicated that the degree of viral suppression while on therapy was more profound with the combination than with either component alone. The largest limitation of those studies, in our opinion, is that the NUCs were discontinued only after a year of treatment.

More recently, some pilot studies have explored potential ways to improve the combination regimen. Chan et al. ${ }^{7}$ have examined various timing in combining p-IFN with lamivudine. They found that simultaneous commencement of both agents was superior, although marginally, to staggered administration. We are aware of three other pilot studies employing the combination between p-IFN and entecavir. Although the design of the studies was heterogeneous and different from ours, they reported seroconversion rates as high as 77\%. ${ }^{17-19}$ Our data corroborate those data that the combination between peg-IFN and a modern NUC leads to a substantially higher rate of serological response than what is expected of the current standard of care.

In addition to the higher incidence of HBe seroconversion, there may be qualitative advantage to the combination therapy. HBe seroconversion has been considered one of the meaningful 
landmarks in the course of patients with chronic HBV infection, because it often represents inactivation of the virus and stabilization of hepatitis activities. However, emerging data suggest that HBe seroconversion following NUC therapy, especially in Asian patients, may not be durable..$^{20,21}$ "Consolidation" treatment with oral agents for at least 6 months after seroconversion may improve the durability and is recommended widely, although the quality of data in support is not very high. ${ }^{22,23}$ Durability of response following IFN therapy may be better., ${ }^{5,624}$ In this small series with modest follow-up, we did not encounter HBeAg sero-reversion, even in the three patients in whom HBV DNA became detectable again. While the long-term outcome in our patients remains to be determined, their response, given their exposure to IFN, may be more durable than patients treated with a NUC alone.

The two adolescents who have not achieved HBe seroconversion might point to a difference in immunological status between adults and adolescents. Adolescents with chronic hepatitis are closer to the immune tolerant phase of HBV infection chronologically and, presumably, biologically. ${ }^{25}$ Of the two adolescents in this series, one has not had a serological response in spite of adequate viral suppression, whereas the other achieved HBs seroconversion without HBe seroconversion. Longer term treatment and follow-up are needed and planned.

There is a prevailing belief that IFN therapy is better tolerated in patients with HBV than those with HCV, which may be attributable to the lack of concomitant ribavirin and/or underlying psychosocial comorbidity commonly present in HCV patients that may reduce tolerance to IFN. In this series, three patients experienced adverse effects of p-IFN that led to its discontinuation. In one patient (case no. 6), p-IFN was discontinued when he had complaints of mild fatigue 24 week after he had already undergone HBe seroconversion. In the other two patients (case no. 7 and 8), p-IFN was discontinued before $\mathrm{HBe}$ seroconversion (at 34 and 16 week, respectively). Both patients eventually seroconverted off p-IFN. Early HBe seroconversion before scheduled p-IFN treatment course (i.e., 48 weeks) in cases 6 along with cases 4 and 5 (at week 8 and 37, respectively), point to the uncertainty about the optimal duration of p-IFN therapy. When used alone, p-IFN therapy for 48 weeks has been shown to be superior to treatment given for 24 weeks. ${ }^{26}$ In the setting of combination therapy with a NUC, what may constitute the optimal use of p-IFN remains to be defined.

Clearly, there are several important limitations to this study. First, as pointed out throughout the manuscript, the sample size is very small and our provocative data are to be construed as pilot case series. Second, the favorable response in our patients may in part be due to patient selection. It is well recognized that HBe seroconversion is more likely in patients with active liver disease as demonstrated by high serum ALT levels and liver histology. Our patients did have evidence for active liver disease, but they also had high HBV DNA, the latter being as- sociated with lower responsive to treatment. Along the same vein, viral genotype may affect serological response to IFN therapy. Unfortunately, genotype data collection was not part of the protocol. Finally, our combination regimen was selected arbitrarily, particularly with regard to the 8 week lead-in with p-IFN. Implementation of our protocol predated publication of the Chan paper which advocated simultaneous commencement of both agents. ${ }^{27}$ Although our data are not directly comparable to theirs because of differences in other aspects of the regimens, such as the choice of NUCs and duration of therapy, our protocol has recently been modified to incorporate simultaneous introduction of the agents for future patients. Finally, we are unable to determine whether entecavir or tenofovir is preferable as a component in combination with p-IFN. Based on prevalent data on monotherapy, we believe it is unlikely that either is significantly better than the other.

In summary, this exploratory study suggests that the combination regimen incorporating $\mathrm{p}$-IFN and a modern oral antiviral agent may be superior to monotherapy with either class of agents alone in the treatment of HBeAg-positive chronic hepatitis B patients. These data urge a randomized controlled trial to properly address the hypothesis with adequate statistical power.

\section{CONFLICTS OF INTEREST}

No potential conflict of interest relevant to this article was reported.

\section{ACKNOWLEDGEMENTS}

Guarantor of the article: The guarantor of this work and its integrity is Dr. W. Ray Kim.

Author contributions: Study concept and design (Dr. Wi, Dr. Kim, Dr. Gross, Dr. Poterucha); acquisition data (Dr. Wi, Ms. Stadheim); analysis and interpretation of data (Dr. Wi, Dr. Kim); drafting of the manuscript (Dr. Wi, Dr. Kim, Ms. Stadheim), critical revision of the manuscript for important intellectual content (Dr. Kim, Dr. Gross, Dr. Poterucha), statistical analysis (Dr. Wi, Dr. Kim).

\section{REFERENCES}

1. Shepard CW, Simard EP, Finelli L, Fiore AE, Bell BP. Hepatitis $B$ virus infection: epidemiology and vaccination. Epidemiol Rev 2006;28:112-125.

2. Chang TT, Liaw YF, Wu SS, et al. Long-term entecavir therapy results in the reversal of fibrosis/cirrhosis and continued histological improvement in patients with chronic hepatitis B. Hepatology 2010;52:886-893.

3. Marcellin P, Gane E, Buti M, et al. Regression of cirrhosis during treatment with tenofovir disoproxil fumarate for chronic hepatitis B: a 5-year open-label follow-up study. Lancet 2013;381:468-475. 
4. Tenney DJ, Rose RE, Baldick CJ, et al. Long-term monitoring shows hepatitis B virus resistance to entecavir in nucleoside-naive patients is rare through 5 years of therapy. Hepatology 2009; 49:1503-1514.

5. Buster EH, Flink HJ, Cakaloglu Y, et al. Sustained HBeAg and HBsAg loss after long-term follow-up of HBeAg-positive patients treated with peginterferon alpha-2b. Gastroenterology 2008;135:459-467.

6. van Zonneveld M, Honkoop P, Hansen BE, et al. Long-term follow-up of alpha-interferon treatment of patients with chronic hepatitis B. Hepatology 2004;39:804-810.

7. Chan HL, Leung NW, Hui AY, et al. A randomized, controlled trial of combination therapy for chronic hepatitis B: comparing pegylated interferon-alpha2b and lamivudine with lamivudine alone. Ann Intern Med 2005;142:240-250.

8. Lau GK, Piratvisuth T, Luo KX, et al. Peginterferon Alfa-2a, lamivudine, and the combination for HBeAg-positive chronic hepatitis B. N Engl J Med 2005;352:2682-2695.

9. Janssen HL, van Zonneveld M, Senturk H, et al. Pegylated interferon alfa-2b alone or in combination with lamivudine for HBeAg-positive chronic hepatitis B: a randomised trial. Lancet 2005;365:123-129.

10. Terrault NA. Benefits and risks of combination therapy for hepatitis B. Hepatology 2009;49:S122-S128.

11. Bedossa P, Poynard T. An algorithm for the grading of activity in chronic hepatitis C. The METAVIR Cooperative Study Group. Hepatology 1996;24:289-293.

12. The French METAVIR Cooperative Study Group. Intraobserver and interobserver variations in liver biopsy interpretation in patients with chronic hepatitis C. Hepatology 1994;20:15-20.

13. Perrillo R. Benefits and risks of interferon therapy for hepatitis B. Hepatology 2009;49:S103-S111.

14. European Association for the Study of the Liver. EASL clinical practice guidelines: management of chronic hepatitis B virus infection. J Hepatol 2012;57:167-185.

15. Lok AS, McMahon BJ. Chronic hepatitis B: update 2009. Hepatology 2009;50:661-662.

16. Woo G, Tomlinson G, Nishikawa Y, et al. Tenofovir and entecavir are the most effective antiviral agents for chronic hepatitis B: a systematic review and Bayesian meta-analyses. Gastroenterology 2010;139:1218-1229.
17. Hagiwara S, Kudo M, Osaki Y, et al. Impact of peginterferon alpha- $2 \mathrm{~b}$ and entecavir hydrate combination therapy on persistent viral suppression in patients with chronic hepatitis B. J Med Virol 2013;85:987-995.

18. Boglione L, D'Avolio A, Cariti G, et al. Sequential therapy with entecavir and PEG-INF in patients affected by chronic hepatitis $B$ and high levels of HBV-DNA with non-D genotypes. J Viral Hepat 2013;20:e11-e19.

19. Enomoto M, Nishiguchi S, Tamori A, et al. Entecavir and interferon-alfa sequential therapy in Japanese patients with hepatitis B e antigen-positive chronic hepatitis B. J Gastroenterol 2013;48:397404.

20. Ryu SH, Chung YH, Choi MH, et al. Long-term additional lamivudine therapy enhances durability of lamivudine-induced HBeAg loss: a prospective study. J Hepatol 2003;39:614-619.

21. Yuen MF, Hui CK, Cheng CC, Wu CH, Lai YP, Lai CL. Long-term follow-up of interferon alfa treatment in Chinese patients with chronic hepatitis B infection: the effect on hepatitis B e antigen seroconversion and the development of cirrhosis-related complications. Hepatology 2001;34:139-145.

22. Dienstag JL, Schiff ER, Mitchell M, et al. Extended lamivudine retreatment for chronic hepatitis B: maintenance of viral suppression after discontinuation of therapy. Hepatology 1999;30:10821087.

23. Dienstag JL, Cianciara J, Karayalcin S, et al. Durability of serologic response after lamivudine treatment of chronic hepatitis B. Hepatology 2003;37:748-755.

24. Lau DT, Everhart J, Kleiner DE, et al. Long-term follow-up of patients with chronic hepatitis B treated with interferon alfa. Gastroenterology 1997;113:1660-1667.

25. Shah U, Kelly D, Chang MH, et al. Management of chronic hepatitis B in children. J Pediatr Gastroenterol Nutr 2009;48:399-404.

26. Liaw YF, Jia JD, Chan HL, et al. Shorter durations and lower doses of peginterferon alfa-2a are associated with inferior hepatitis B e antigen seroconversion rates in hepatitis B virus genotypes B or C. Hepatology 2011;54:1591-1599.

27. Chan HL, Wong VW, Chim AM, et al. Virological response to different combination regimes of peginterferon alpha-2b and lamivudine in hepatitis B e antigen positive chronic hepatitis B. Antivir Ther 2007;12:815-823. 